Adriana Gomes LUZ ${ }^{1}$

Douglas Bernal Tiago ${ }^{2}$

José Carlos Gama da Silva ${ }^{3}$

Eliana AMARAL ${ }^{4}$

Artigos originais

Palavras-chave

Morbidade

Gestação de alto risco

Mortalidade materna/epidemiologia

Fatores de risco

Complicações na gravidez

Keywords

Morbidity

Pregnancy, high risk

Maternal mortality/epidemiology

Risk factors

Pregnancy complications

\section{Morbidade materna grave em um hospital universitário de referência municipal em Campinas, Estado de São Paulo}

\author{
Severe maternal morbidity at a local reference university bospital \\ in Campinas, São Paulo, Brazil
}

\section{Resumo}

OBJETIVO: avaliar a prevalência e os fatores de risco associados à morbidade materna grave numa maternidade terciária. MÉTODOS: trata-se de um estudo de corte transversal dos casos de morbidade materna grave atendidos no Hospital e Maternidade Celso Pierro entre outubro de 2005 e julho de 2006, identificados a partir dos livros de controle das unidades de internação, pronto atendimento e centro obstétrico. Foram estudadas gestantes e puérperas que apresentavam condições definidoras de morbidade materna grave a partir dos diagnósticos clínicos segundo critérios propostos por Waterstone. Posteriormente, os casos de maior gravidade clínica, chamados de morbidade extremamente grave, foram reclassificados utilizando-se os critérios definidores de Mantel, baseados em disfunção orgânica e manejo. RESULTADOS: foram identificadas 114 mulheres com morbidade materna grave entre 2.207 partos, com razão de outra morbidade grave e morbidade extremamente grave de 44,9 e 6,8 casos por 1.000 partos, respectivamente. A média da idade gestacional no parto foi de 35 semanas e $87 \%$ das mulheres vieram de área de cobertura da maternidade no município. A hipertensão (pré-eclâmpsia grave) representou $96 \%$ de outras morbidades graves e a hemorragia esteve presente em $60 \%$ dos casos de morbidade extremamente grave, seguida de hipertensão. A prevalência de morbidade extremamente grave entre os casos de morbidade grave não se associou com estado marital, escolaridade, idade materna, tipo de parto, gestações, idade gestacional e distrito de saúde de moradia. CONCLUSÕES: as outras morbidades graves foram 6,6 vezes mais freqüentes que os casos extremamente graves, sem diferenças entre os grupos por fatores de risco epidemiológicos.

\section{Abstract}

PURPOSE: to assess the prevalence and risk factors associated with near miss and other severe maternal morbidity at a reference tertiary maternity. METHODS: this is a cross-sectional study on severe maternal morbidity at the Hospital e Maternidade Celso Pierro, Campinas, São Paulo, between October 2005 and July 2006, identified from infirmary, admission and delivery unit logbooks. Pregnant and post-partum women with severe maternal morbidity were identified according to clinical criteria proposed by Waterstone. Later, cases with more severe morbidity, called extremely severe maternal morbidity, were reclassified using Mantel criteria, based on organic dysfunction and clinical management. RESULTS: there were 114 severe maternal morbidity cases among 2,207 birth deliveries, with a ratio of other severe morbidity and extremely severe morbidity near miss of 44.9 and 6.8 cases/ 1,000 live births, respectively. Mean gestational age at delivery was 35 weeks, and $87 \%$ came from the reference area for the maternity service. Hypertension (severe pre-eclampsia) represented $96 \%$ of other severe morbidity, while hemorrhage represented $60 \%$ of all extremely severe cases, followed by hypertension. The prevalence of extremely severe morbidity among the severe morbidity cases was not associated with marital status, schooling, maternal age, type of delivery, parity, gestational age at birth and home place. CONCLUSIONS: the other morbidities were 6.6 times more frequent than near miss, and it was not possible to differentiate both groups by epidemiological risk factors
Correspondência:

Adriana Gomes Luz Rua Barreto Leme, 1.878 - Cambuí CEP 13025-085 - Campinas/SP Fone/Fax: (19) 3251-8399/3294-1655 E-mail: adrigoluz@terra.com.br

Recebido

07/01/2008

Aceito com modificacões

$03 / 06 / 2008$
Hospital e Maternidade Celso Pierro da Pontifícia Universidade Católica de Campinas - PUC-Campinas - Campinas (SP), Brasil. 'Pós-graduanda do Departamento de Tocoginecologia da Faculdade de Ciências Médicas da Universidade Estadual de Campinas - UNICAMP- Campinas (SP), Brasil; Médica Assistente do Hospital e Maternidade Celso Pierro da Pontifícia Universidade Católica de Campinas -PUC-Campinas - Campinas (SP), Brasil.

2 Professor Adjunto do Departamento de Tocoginecologia da Pontifícia Universidade Católica de Campinas - PUC-Campinas Campinas (SP), Brasil.

${ }^{3}$ Professor Titular do Departamento de Tocoginecologia da Pontifícia Universidade Católica de Campinas - PUC-Campinas - Campinas (SP), Brasil.

4 Professora Associada do Departamento de Tocoginecologia da Faculdade de Ciências Médicas da Universidade Estadual de Campinas - UNICAMP - Campinas (SP), Brasil. 


\section{Introdução}

Em 2004, a Organização Mundial da Saúde (OMS) estimou que, além da ocorrência de cerca de 529.000 óbitos maternos, 20 milhões de mulheres tenham apresentado complicações agudas da gestação, com considerável número de seqüelas ${ }^{1}$. Entretanto, enquanto a razão de morte materna (RMM) é de 20/100.000 nascidos vivos nas regiões desenvolvidas, esta atinge 440/100.000 nas regiões em desenvolvimento, sendo 190 na América Latina e Caribe ${ }^{1}$.

No Brasil, onde a RMM oficial é de aproximadamente 76/100.000 nascidos vivos, podem estar ocorrendo até cem vezes mais casos de mulheres com morbidade grave $(7.600 / 100.000 \text { ou } 7,6 \%)^{2,3}$. Num estudo em capitais brasileiras, $67,1 \%$ das mortes maternas foram decorrentes de causas obstétricas diretas. Predominaram os transtornos hipertensivos em todas as mortes, presentes em $25 \%$ dos casos; a pré-eclâmpsia e a eclâmpsia responderam por $37 \%$ das mortes obstétricas diretas. As complicações hemorrágicas, particularmente devidas à placenta prévia e ao descolamento prematuro da placenta, foram responsáveis por 9 do total e 13,3\% das mortes obstétricas diretas. Entretanto, cerca de um quarto das mortes foram por causas obstétricas indiretas ${ }^{4}$. Esses dados indicam que a qualificação da assistência nas instituições brasileiras deve enfocar tanto as causas de morte diretamente relacionadas à gestação quanto as condições clínicas prévias à gestação, com especial atenção ao cuidado no hospital, onde ocorrem 90\% dos partos ${ }^{3}$.

Tem crescido o interesse no estudo dos casos de morbidade grave, particularmente casos de morbidade extremamente grave (near miss). A morbidade materna é um continuum que se inicia com a ocorrência de uma complicação durante a gestação, parto ou puerpério, e que pode terminar em morte. Os casos de morbidade extremamente grave são aqueles em que mulheres apresentam complicações potencialmente fatais durante a gravidez, parto ou puerpério, mas sobrevivem devido ao acaso ou ao bom cuidado hospitalar ${ }^{5-7}$.

Como a sobrevivência da mulher depende, muitas vezes, do cuidado oferecido pelo serviço, o estudo pormenorizado destes casos tem sido utilizado para avaliar não apenas a incidência de complicações ameaçadoras da vida, como também a qualidade na atenção obstétrica ${ }^{8,9}$. Reconhecer os casos críticos de urgências e emergências maternas também oferece a oportunidade de intervir a tempo, evitando uma morte potencial ${ }^{1}$. Além disso, a revisão dos casos críticos permite a identificação dos fatores responsáveis pelo seu desencadeamento, favorecendo a implantação posterior de ações preventivas capazes de interferir no processo evolutivo das complicações que podem levar à morte.

A falta de um planejamento detalhado para a oferta de cuidados pode favorecer a ocorrência de demoras na implantação de medidas necessárias ${ }^{10,11}$ e uma forma de reduzir o risco de morte materna é pela melhoria no atendimento à gestante. As complicações obstétricas graves podem ser eventos sentinelas úteis nas revisões dos casos para vigilância ou auditorias ${ }^{12}$.

Entretanto, a delimitação entre as situações clínicas de morbidade extremamente grave e outros casos de morbidade grave é polêmica. Alguns autores utilizam definições muito amplas que incluem situações clínicas menos severas. Há diferentes critérios definidores de morbidade materna extremamente grave, baseados em manejo em Unidade de Terapia Intensiva (UTI), sinais e sintomas clínicos e disfunção orgânica ${ }^{9,12}$. Como a disponibilidade e utilização de leitos de UTI não são uniformes, as definições baseadas em sinais e sintomas clínicos e na evidência de disfunção orgânica oferecem a possibilidade de uma definição mais homogênea.

O objetivo do estudo foi avaliar a taxa de ocorrência, condições definidoras e os fatores de riscos associados à morbidade extremamente grave, comparando a outras situações de morbidade materna grave num hospital universitário, que responde pelo atendimento a uma população de baixo poder aquisitivo, majoritariamente dependente do Sistema Único de Saúde (SUS), de um grande centro urbano da região sudeste do Brasil.

\section{Métodos}

Este estudo de corte transversal foi realizado entre outubro de 2005 e julho de 2006 no Hospital e Maternidade Celso Pierro (HMCP), da Pontifícia Universidade Católica de Campinas (PUC-Campinas), que atende majoritariamente partos da população dependente do SUS dentro do município de Campinas, São Paulo, cuja área de abrangência inclui 375.500 habitantes da cidade ${ }^{13}$. Trata-se de uma instituição de nível terciário de atenção, equipado com centro cirúrgico e centro obstétrico, UTI para adultos, UTI neonatal e cardiológica, com equipes médicas de plantão 24 horas - incluindo obstetra, anestesista, cardiologista, pediatra, intensivista, cirurgião geral e outras especialidades, em caso de necessidade.

Os casos foram identificados diariamente por uma enfermeira capacitada, e os dados foram coletados pela pesquisadora nos prontuários clínicos durante e após as altas hospitalares e transcritos para ficha de coleta sem contato direto ou entrevista com as mulheres. O estudo foi aprovado pelo Comitê de Ética em Pesquisa da PUC - Campinas.

Foram estudadas gestantes e puérperas que apresentavam condições definidoras de morbidade materna grave a partir dos diagnósticos clínicos das fichas de pronto atendimento e livro de registro de internação hospitalar da Enfermaria de Obstetrícia e Centro Obstétrico, seguindo 
os critérios clínicos de Waterstone, Bewley e Wolfe apresentados no Quadro 1. Posteriormente, os mesmos casos foram reclassificados pelos critérios de Mantel et al. ${ }^{14}$, que identificam situações de maior gravidade clínica, chamados de morbidade extremamente grave (Quadro 2). Os casos de hemorragia na morbidade extremamente grave foram identificados como necessidade de transfusão de três ou mais concentrados de hemácias (perda sanguínea $>1.500 \mathrm{~mL})^{9}$. Assim, alguns casos de morbidade grave que não preencheram os critérios de Mantel et al. ${ }^{14}$ foram considerados de menor gravidade dentro do espectro, compondo o grupo identificado como outras morbidades graves.

O cálculo do tamanho da amostra foi baseado na prevalência de morbidade materna grave em hospital do mesmo município, de 42 casos por 1.000 partos $(4,2 \%)^{15}$. Assumindo-se um nível de significância de $5 \%$ e um erro amostral de $1 \%$, estimou-se que seria necessário avaliar 1.546 mulheres num período de 12 meses para que houvesse, no mínimo, 3,2\% casos de morbidade materna grave ou 32 mulheres a cada 1.000 partos.

Para análise estatística, calculou-se a razão de prevalência e o intervalo de confiança de $95 \%$ para a ocorrência de morbidade materna extremamente grave e das outras condições clínicas de morbidade grave. Procuraram-se diferenciar os dois grupos de morbidade segundo alguns fatores de risco sociodemográficos, incluindo estado marital, escolaridade, idade materna, tipo de parto, número de gestações, idade gestacional e distrito de saúde de moradia no município.

\section{Resultados}

No período estudado, 2.207 mulheres tiveram parto na instituição. Entre estas, foram identificadas $114 \mathrm{com}$ morbidade materna grave. Deste grupo, 15 casos foram re-classificados como morbidade extremamente grave e 99 casos como outras morbidades graves, ou um caso de morbidade extremamente grave para sete casos das outras morbidades. Assim, foi observada uma razão de 6,8 por 1.000 partos de morbidade extremamente grave e 44,9 casos por 1.000 partos de outras morbidades (Tabela 1).

A maioria dos casos de morbidade materna extremamente grave deveu-se à hemorragia pós-parto, tendo ocorrido também quatro casos de internação em UTI, dois casos de histerectomia pós-parto, um de edema agudo de pulmão, um de sepse e uma mulher com crise tireotóxica. A hipertensão foi o fator determinante quase exclusivo dos casos de outras formas de morbidade grave (Tabela 1). Houve um óbito materno no mesmo período, devido à sepse puerperal.

A média da idade gestacional no parto foi de 35 semanas nos dois grupos e $87 \%$ das mulheres vieram de área de cobertura da maternidade dentro do município. Pouco mais de $50 \%$ das mulheres eram unidas (casadas ou amasiadas) e quase metade delas tinha apenas uma gestação. A maioria apresentava idade superior a 20 anos, com mais de oito anos de estudos e tiveram parto cesáreo. A prevalência de morbidade extremamente grave entre os casos de morbidade grave não esteve associada a estado marital, escolaridade, idade materna, tipo de parto, número de gestações, idade gestacional e distrito de saúde de moradia (Tabela 2).

Quadro 1 - Critérios diagnósticos de morbidade materna grave segundo Waterstone, Bewley, Wolfe ${ }^{6}$

1. Pré-eclâmpsia grave 0
$P A=170 / 110 \mathrm{mmHg}, 2$ vezes com 4 horas de intervalo ou
$P A>170 / 110$ em uma medida associada à proteinúria 24 horas maior que $0,3 \mathrm{~g}$ ou ++ em fita
2. Eclâmpsia
3. Síndrome Hellp
4. Hemorragia grave (perda sanguínea $>1.500 \mathrm{~mL}$ )
5. Sepse grave
6. Rotura uterina

Quadro 2 - Critérios diagnósticos de morbidade extremamente grave (near miss) segundo Mantel et al. ${ }^{14}$

\begin{tabular}{|c|}
\hline 1. Edema pulmonar \\
\hline 2. Parada cardiorrespiratória \\
\hline 3. Hipovolemia (necessidade de 5 ou mais unidades de concentrado de hemáceas) \\
\hline 4. Admissão à Unidade de Terapia Intensiva (UTI) por sepse ou outra causa \\
\hline 5. Histerectomia de emergência \\
\hline 6. Ventilação por mais de 60 minutos, exceto em anestesia geral \\
\hline 7. Saturação de $\mathrm{O}_{2}$ inferior a $90 \%$, por mais de 60 minutos \\
\hline $\begin{array}{l}\text { 8. Relaccão pressão alveolar de } \mathrm{O}_{2} / \text { fraçãa inspirada de } \mathrm{O}_{2}\left(\mathrm{PaO}_{2} / \mathrm{FiO}_{2}\right)>300 \mathrm{mmHg} \\
\text { 9. Diurese inferior a } 400 \mathrm{~mL} / 24 \text { horas, refratária a hidrataç̦ẫo, furosemida ou dopamina }\end{array}$ \\
\hline 10. Deterioração aguda dos níveis de uréia e creatinina ( $>15 \mathrm{~mol}$ e $>400 \mathrm{~mol}$ ) \\
\hline 11. Icterícia na presença de pré-eclâmpsia \\
\hline 12. Cetoacidose diabética \\
\hline 13. Crise tireotóxica \\
\hline 14. Trombocitopenia aguda requerendo transfusão de plaquetas \\
\hline 15. Coma por mais de 12 horas \\
\hline $\begin{array}{l}\text { 16. Hemorragia subaracnóide ou intraparenquimatosa } \\
\text { 17. Acidente anestésico: hipotensão grave pós-bloqueio e insucesso de intubação }\end{array}$ \\
\hline
\end{tabular}

Tabela 1 - Condições identificadoras associadas aos casos de morbidade materna grave

\begin{tabular}{|c|c|c|c|c|}
\hline \multirow[t]{2}{*}{$\begin{array}{l}\text { Condições identificadoras de } \\
\text { morbidade materna grave }\end{array}$} & \multicolumn{2}{|c|}{$\begin{array}{c}\text { Morbidade } \\
\text { extremamente grave }\end{array}$} & \multicolumn{2}{|c|}{$\begin{array}{c}\text { Outras morbidades } \\
\text { graves }\end{array}$} \\
\hline & n & $\%$ & n & $\%$ \\
\hline Hipertensão" & 2 & 13,3 & 95 & 96,0 \\
\hline Hemorragia anteparto" & 2 & 13,3 & 1 & 1,0 \\
\hline Hemorragia pós-parto\# & 7 & 46,7 & 3 & 3,0 \\
\hline Sepse puerperal & 1 & 6,7 & - & - \\
\hline Insuficiência respiratória* & 2 & 13,4 & - & - \\
\hline Insuficiência renal crônica* & 1 & 6,7 & - & - \\
\hline Total & 15 & 100,0 & 99 & 100,0 \\
\hline Razão/1.000 partos & 6,8 & - & 44,9 & - \\
\hline
\end{tabular}

"Hipertensão $x$ hemorragia, $\mathrm{p}<0,0001$ (teste de Fisher); *não incluídos pelos critérios de Waterstone. 
Tabela 2 - Características gerais das pacientes, origem geográfica e gravidez e a associação com morbidade materna grave

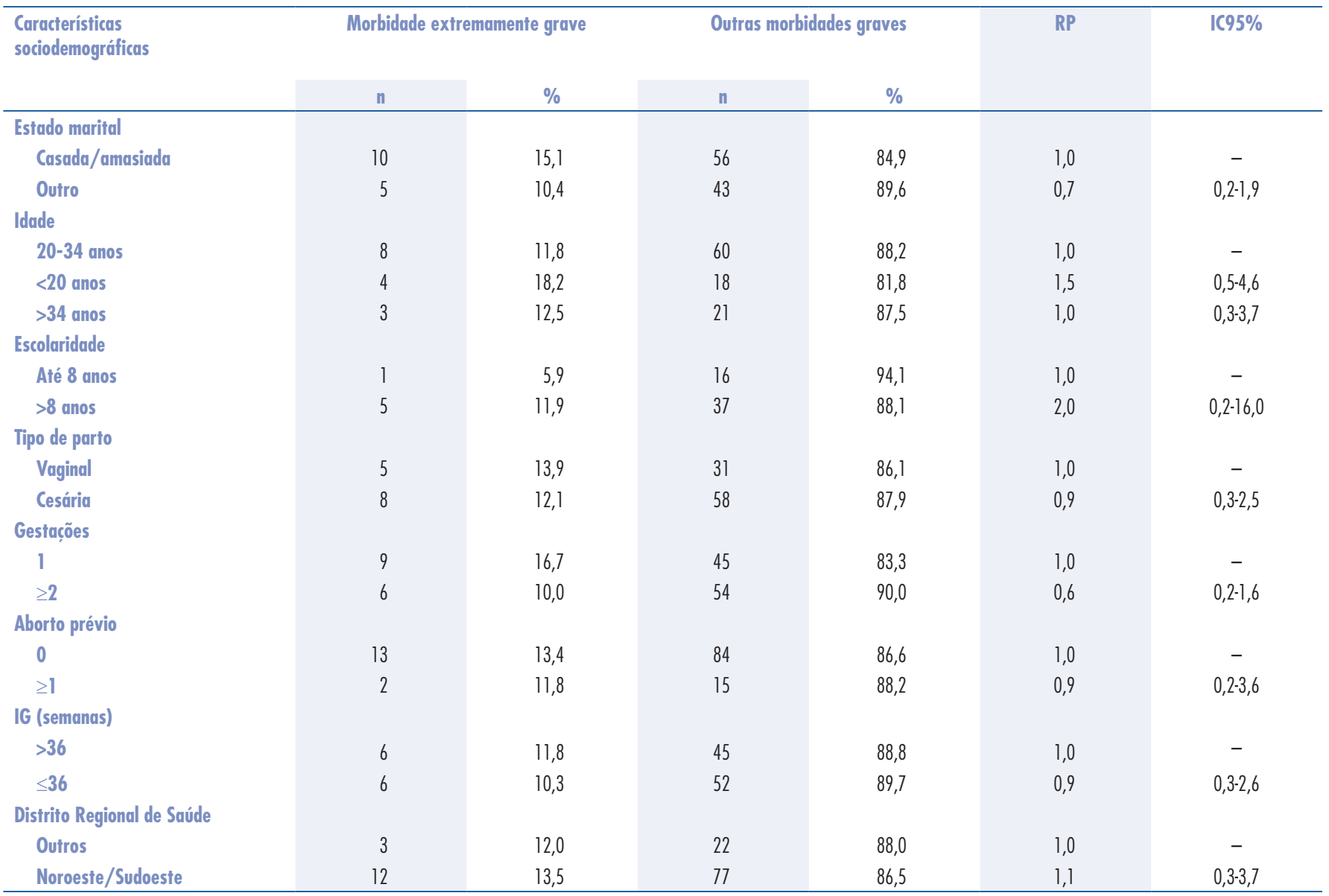

\section{Discussão}

Os resultados mostraram que, por critérios de disfunção orgânica e manejo clínico, houve 15 casos de morbidade extremamente grave ou near miss, correspondendo a 6,8 casos por 1.000 partos. Estas situações clínicas mais graves correspondem a $13 \%$ do total de casos de morbidade identificados e derivaram, em grande proporção, de hemorragia puerperal. Mas a razão de morbidade materna grave total, decorrente do somatório dos casos de extrema gravidade e outros menos severos, foi de 51,7 casos por 1.000 partos. Portanto, até $5 \%$ dos casos atendidos nesta maternidade de referência, que dá cobertura a uma população com os piores Índices de Condição de Vida (ICV) ${ }^{16}$ do município, podem ter uma condição suficientemente grave para merecer atenção especializada, sendo que um pouco menos de $1 \%$ necessita de cuidados compatíveis com atendimento em UTI. Como em outras populações, os diagnósticos mais comumente associados à morbidade extremamente grave foram hemorragia e hipertensão ${ }^{17}$. Isso significa que a qualificação na prevenção e tratamento destas duas condições deveria ser a prioridade para a equipe desta instituição.
A população desta amostra tem um perfil diferente da população-fonte dos casos estudados por Souza et al. ${ }^{15} \mathrm{em}$ outra instituição universitária de Campinas. Esta última é referência terciária regional para 3.000.000 de habitantes de um conjunto de 42 municípios. No entanto, encontra-se a mesma razão de morbidade extremamente grave, utilizando os mesmos critérios. Isto sugere que as diferenças populacionais não interferem nas taxas de ocorrência das situações clínicas mais severas. Também é possível que haja uma relativa homogeneidade na qualidade da assistência à gestação e ao parto entre os municípios da região.

Por outro lado, estes dois estudos apresentaram a hemorragia como a condição mais prevalente nos casos de morbidade extremamente grave, o que também foi a principal causa de morbidade extremamente grave em estudos africanos ${ }^{6,18}$. Tal fato mostra a urgência de capacitar o pessoal no manejo da profilaxia e tratamento de condições hemorrágicas. Como se estima que apenas $0,03 \%$ das mulheres tenham perda por sangramento maiores que $1.500 \mathrm{~mL}$ no parto, a importância relativa da hemorragia torna-se ainda mais preocupante ${ }^{19}$.

A hipertensão foi causa quase exclusiva de outras morbidades graves, como em outros estudos, sendo causa 
de $47,5 \%$ de outras morbidades graves e $18,2 \%$ das morbidades extremamente graves ${ }^{20}$. Assim, as síndromes hipertensivas são outra demanda primordial para capacitação das equipes obstétricas.

Mas a falta de um consenso na literatura internacional para definição dos casos de morbidade extremamente grave é um dos grandes desafios para sua utilização como evento-gatilho para auditoria ou evento-sentinela para vigilância, objetivando melhorar a qualidade da atenção obstétrica. A inexistência de uma definição operacional homogênea acarreta discrepâncias que prejudicam as conclusões baseadas na melhores evidências disponíveis, até mesmo na quantificação do problema da morbidade materna grave e de seus casos mais extremos ${ }^{19,21,22}$.

Os critérios de disfunção orgânica e manejo, propostos por Mantel et al. ${ }^{14}$, apesar de serem mais objetivos, excluem uma entidade clínica que afetou uma parcela importante das mulheres com outras morbidades graves do nosso estudo, que são as síndromes hipertensivas. Pelos critérios de Mantel et al. ${ }^{14}$, a hipertensão só é considerada quando ocorrem complicações gravíssimas, sinalizadas por icterícia e trombocitopenia aguda com necessidade de transfusão de plaquetas - ocorrência rara no extremo do espectro clínico destas condições. Entretanto, somos da opinião, juntamente com outros autores, que os casos mais graves de hipertensão, como eclâmpsia e síndrome Hellp, também devem ser incluídos na definição de morbidade extremamente grave ou near miss ${ }^{19,21}$.

O critério de anemia severa de Mantel et al. ${ }^{14}$ (mais do que cinco unidades de transfusão) é tão rigoroso que outros autores o modificaram em seus estudos; Geller et al. ${ }^{21}$ consideraram perda sanguínea importante aquela maior que $1.500 \mathrm{~mL}$ ou três unidades de hemácias transfundidas. Se fosse utilizado o critério de cinco unidades de transfusão, todos os casos de hemorragia teriam sido excluídos da categoria de morbidade extremamente grave. Assim, é necessário adotar critérios definidores de morbidade extremamente grave que não sejam tão restritivos quanto aqueles propostos por Mantel et al. ${ }^{14}$ e um pouco mais seletivos do que os critérios de Waterstone, Bewley e Wolfe ${ }^{6}$.
Uma limitação deste estudo foi a carência de dados mais qualificados sobre histórico do acompanhamento pré-natal e algumas características sociodemográficas, que poderiam ser obtidos com a entrevista da mulher, mesmo durante a internação. Entretanto, não foi proposto incluir variáveis identificadoras das situações clínicas de gravidade que dependessem de entrevista, porque eram procurados dados de fácil acesso que pudessem ser reproduzidos em outras instituições. Ficou evidente a dificuldade de se obter informação sobre escolaridade, idade gestacional no início do pré-natal e número de consultas de pré-natal, que deveria estar registrada nos prontuários clínicos. Esta constatação, de má qualidade dos registros essenciais sociodemográficos, já confirmou a necessidade de educação continuada dos profissionais para qualificar as informações dos prontuários clínicos.

Se a principal razão para se identificar os casos de morbidade materna extremamente grave é poder intervir a tempo de evitar a evolução para morte, é necessário encontrar o ponto de equilíbrio entre critérios muito específicos, pouco sensíveis, mas que permitam atuação clínica a tempo de salvaguardar a saúde materna. Atingir este equilíbrio é fundamental não apenas para uma adequada prevenção das mortes, como também para evitar a inclusão de muitos casos falsos-positivos num processo de vigilância dos casos de morbidade grave ou extremamente grave, que tornasse pouco operacional sua utilização como intervenção para qualificar a atenção obstétrica.

Em conclusão, o estudo da morbidade materna extremamente grave e de outras morbidades nesta maternidade de referência de um grande centro urbano pode contribuir para dimensionar o problema, além de identificar as condições clínicas mais relevantes a elas associadas, o que é essencial na formulação de estratégias para reduzir a morbimortalidade materna.

\section{Agradecimentos}

Ao professor doutor José Guilherme Cecatti pelos comentários e sugestões na discussão dos resultados e preparação da primeira versão deste artigo.

\section{Referências}

1. World Health Organization. Beyond the numbers: reviewing maternal death and complications to make pregnancy safer [ Internet]. Geneva: WHO 2004 [cited 2007 Jun 20]. Available from: http://www. who.int/reproductive-health/publications/btn/text.pdf
2. Ministério da Saúde. Indicadores de mortalidade. C.3 Razão de mortalidade materna [Internet]. IDB; 2006 [citado 21 Jun 2007]. Disponível em: http://tabnet.datasus.gov.br/cgi/ idb2006/c03.htm 
3. Laurenti R, Jorge MHPM, Gotlieb SLD. A mortalidade materna nas capitais brasileiras: algumas características e estimativas de um fator de ajuste. Rev Bras Epidemiol. 2004;7(4):449-60.

4. Ministério da Saúde. Secretaria de Atenção à Saúde. Estudo da mortalidade de mulheres de 10 a 49 anos, com ênfase na mortalidade materna. Relatório final [Internet]. Brasília; 2006 [citado 21 Jun 2007]. Disponível em: http://portal.saude.gov. $\mathrm{br} /$ portal/arquivos/pdf/estudo_mortalidade_texto.pdf

5. Amaral E, Luz AG, Souza JPD. A morbidade materna grave na qualificação da assistência: utopia ou necessidade? Rev Bras Ginecol Obstet. 2007;29(9):484-9.

6. Waterstone M, Bewley S, Wolfe C. Incidence and predictors of severe obstetric morbidity: case-control study. BM. 2001;322(7294): 1089-93.

7. Stones W, Lim W, Al-Azzawi F, Kelly M. An investigation of maternal morbidity with identification of life- threatening "near miss" episodes. Health Trends. 1991;23(1):13-5.

8. Pattinson RC, Hall M. Near misses: a useful adjunct to maternal death enquiries. Br Med Bull. 2003;67:231-43.

9. Geller SE, Rosenberg D, Cox SM, Kilpatrick S. Defining a conceptual framework for near-miss maternal morbidity. J Am Med Womens Assoc. 2002;57(3):135-9.

10. Ministério da Saúde. Urgências e emergências maternas: guia para diagnóstico e conduta em situações de risco de morte materna. 2ª ed. Brasília: Ministério de Saúde; 2000.

11. Maine D, Akalin MZ, Ward VM, Kamara A. The design and evaluation of maternal mortality programs. New York: Columbia University/Center for Population and Family Health; 1997.

12. Penney G, Brace V. Near miss audit in obstetrics. Curr Opin Obstet Gynecol. 2007; 19(2): 145-50.

13. Prefeitura Municipal de Campinas. Secretaria Municipal de Saúde. Plano Municipal de Saúde 2006/2007 [Internet]. Campinas; 2006 [citado 12 maio 2007]. Disponível em: http://www.campinas. sp.gov.br/saude

14. Mantel GD, Buchmann E, Rees H, Pattinson RC. Severe acute maternal morbidity: a pilot study of a definition for a near-miss. $\mathrm{Br}$ J Obstet Gynaecol. 1998; 105(9):985-90.

15. Souza JP, Cecatti JG, Parpinelli MA, Serruya SJ, Amaral E. Appropriate criteria for identification of near-miss maternal morbidity in tertiary care facilities: a cross sectional study. BMC Pregnancy Childbirth 2007;7:20.

16. Prefeitura Municipal de Campinas. Secretaria Municipal de Saúde. SPICV - Índice de Condição de Vida [documento da Internet]. 2001 [citado 21 jun 2007]. Disponível em: http://www.campinas. sp.gov.br/saude/dados/icv/icv_index.htm

17. Adisasmita A, Deviany PE, Nandiaty F, Stanton C, Ronsmans C. Obstetric near miss and deaths in public and private hospital in Indonesia. BMC Pregnancy Childbirth. 2008;8:10.

18. Minkauskienė M, Nadišauskienė R, Padaigal Z, Makari S. Systematic review on the incidence and prevalence of severe maternal morbidity. Medicina (Kaunas). 2004;40(4):299-309.

19. Geller SE, Rosenberg D, Cox SM, Brown ML, Simonson L, Driscoll CA, et al. The continuum of maternal morbidity and mortality: factors associated with severity. Am J Obstet Gynecol. 2004; 191(3):939-44.

20. Filippi V, Ronsmans C, Gohou V, Goufodji S, Lardi M, Sahel A, et al. Maternity wards or emergency obstetric rooms? Incidente of near- miss events in African hospitals. Acta Obstet Gynecol Scand. 2005;84(1):11-6.

21. Geller SE, Rosenberg D, Cox SM, Brown M, Simonson L, Kilpatrick S. A scoring system identified near-miss maternal morbidity during pregnancy. J Clin Epidemiol. 2004;57(7):716-20.

22. Cecatti JG, Souza JP, Parpinelli MA, de Sousa MH, Amaral E. Research on severe maternal morbidities and near-misses in Brazil: what we have learned. Reprod Health Matters. 2007;15(30):125-33. 